\title{
PRIORITÄTSDIENST DES BEHINDERTENSTATUTS UND DES PRIORITÄTSPRINZIPS BEI DER IMMOBILIENANMELDUNG
}

\section{ORIGINALER ARTIKEL}

NETO, Alexandre Moura Lima ${ }^{1}$, AGUAIR, Alessandra Anchieta Moreira Lima de ${ }^{2}$, NETO, Haroldo Corrêa Cavalcanti ${ }^{3}$

NETO, Alexandre Moura Lima. AGUAIR, Alessandra Anchieta Moreira Lima de. NETO, Haroldo Corrêa Cavalcanti. Prioritätsdienst des Behindertenstatuts und des Prioritätsprinzips bei der Immobilienanmeldung. Revista Científica Multidisciplinar Núcleo do Conhecimento. Jahrgang 06, Ed. 09, Vol. 03, S. 45-63. September 2021. ISSN: 2448-0959, Zugangslink: https:/www.nucleodoconhecimento.com.br/gesetz/prioritaetsdienst, DOI: 10.32749/nucleodoconhecimento.com.br/gesetz/prioritaetsdienst

\section{ZUSAMMENFASSUNG}

Die vorliegende Studie zielt darauf ab, den offensichtlichen Konflikt zwischen der vorrangigen Betreuung des Status der Person mit Behinderungen und dem Prioritätsprinzip der Registrierung von Immobilien zu analysieren, dh wenn diese gesetzliche Garantie der Person mit Behinderung bei der Notierung von Rechten in Notatorien Vorrang einricht. In Bezug auf die verwendete Methodik ist es bemerkenswert, dass diese Studie in Bezug auf die Zwecke dieser Studie als beschreibend und erklärend eingestuft wird und, was die Mittel betrifft, als bibliographisch eingestuft wird, wobei Materialien wie Bücher, Artikel, Zeitschriften und Berichte zu diesem Thema verwendet werden. Die Forschung wird auch als qualitativer Ansatz charakterisiert. Es sollte nachgewiesen werden, dass die Person

\footnotetext{
${ }^{1}$ Master in Kultur und Gesellschaft an der Bundesuniversität Maranhão (UFMA). ORCID: https://orcid.org/0000-0002-7232-8449

${ }^{2}$ Er hat einen Master-Abschluss in Umwelt des Graduiertenprogramm in Umwelt der Universität Ceuma. ORCID: https://orcid.org/0000-0003-4017-1579

${ }^{3}$ Spezialisierung im Immobilienrecht. ORCID: https://orcid.org/0000-0003-1746-1965
}

RC: 97618

Verfügbar in: https://www.nucleodoconhecimento.com.br/gesetz/prioritaetsdienst 
mit Behinderung das nach dem Gesetz 13.143/2015 garantierte Recht hat, schnell, effektiv und individuell bedient zu werden, auch im Grundbuchregister, aber keine Privilegien im Hinblick auf die Registrierungspriorität im Hinblick auf den Grundsatz der Registrierungspriorität genießt. Aus den Entscheidungen der eingetragenen Gerichte von São Paulo geht hervor, dass die spezifische Bestimmung des Gesetzes Nr. 6.015/1973 in Bezug auf das Recht auf vorrangige Betreuung bestimmter Personen, gesetzlich 13.143/2015, vorrangig ist, da es sich um ein materielles Prioritätsrecht handelt und davon ausgegangen wird, dass die Gewährung einer vorrangigen Betreuung außerhalb der Anforderungen von Art. 186 des Gesetzes Nr. $6,015 / 1973$ so verstanden wird, dass eine vorrangige Pflege außerhalb der Anforderungen von Art. 186 des Gesetzes Nr. 6,015/1973 gewährt wird. wäre ein unbestreitbarer Affront gegen gesetzliche Diktate, die den Dienst am Recht auf Gleichheit, einer Prämisse auch des Statuts der Menschen mit Behinderungen, gefährden würden.

Schlüsselwörter: Statut der behinderten Person, Registrierung von Immobilien, Prioritätsprinzip.

\section{EINLEITUNG}

Das tägliche Leben von Menschen mit besonderen Bedürfnissen ist komplex, wenn man ihre Grenzen und die mangelnde Vorbereitung der Gesellschaft darauf bedenkt, sie aufzunehmen, sowohl in Bezug auf die Infrastruktur als auch auf die Mentalität des Einzelnen, so dass sie am Ende sozial ausgeschlossen sind, Aktivitäten wie Spaß haben, studieren und zur Arbeit gehen, die einfach sind und Routineaufgaben werden zu einer Herausforderung.

Wie Carlos Henrique Ribeiro da Silva (2008) hervorhebt, führte der Mangel an Toleranz zwischen den Verschiedenen im Laufe der Geschichte dazu, dass Minderheiten immer relativ aggressiv und verwirrt behandelt wurden, indem sie diejenigen, die vom Standard abwichen, der aus einem hegemonialen Modell gebildet wurde, etikettierten, trennten, diskriminierten und ausschlossen. Ein weiterer

$\mathrm{RC}: 97618$

Verfügbar in: https://www.nucleodoconhecimento.com.br/gesetz/prioritaetsdienst 
sehr gemeinsamer Faktor, der durch das mangelnde Verständnis für die Unterschiede zwischen den Menschen entsteht, liegt in der Person, die als anders betrachtet wird, die sehr spezielle Einstellungen wie Selbstbestrafung, Isolation und Aggressivität annimmt. So wird angenommen, dass der Mangel an Toleranz gegenüber den Verschiedenen historische Wurzeln hat, obwohl in Bezug auf das Thema bereits viel fortgeschritten ist.

Lange Zeit galt die Person mit Behinderungen in Brasilien als unfähig, mit den sozialen Veränderungen wurde diese Tatsache geändert und diese Menschen wurden als Subjekte des Rechts anerkannt und konnten ihre Entscheidungen treffen, so sehr, dass im Jahr 2015 das Gesetz Nr. 13.146 das Bürgerliche Gesetzbuch änderte und das Statut der Menschen mit Behinderungen einführte. Laut Lago (2016) zielte die Veröffentlichung dieses Statuts darauf ab, das Wohlergehen und die Überwindung von Barrieren für Menschen mit Behinderungen zu überwinden, als Prämisse, um das Recht auf Würde, Höflichkeit und volle Teilhabe an der Gesellschaft zu gewährleisten. Nach Ansicht des Autors steht die Priorität, die diesen Personen durch das Gesetz eingeräumt wird, jedoch im Widerspruch zum Prioritätsprinzip bei der Registrierung von Immobilien nach dem Gesetz 6.015/1973 und dem Bürgerlichen Gesetzbuch in der Reihenfolge der Präsentation der Titel, die im Mittelpunkt dieser Studie steht. Daher wird es versuchen, in diesem Aufsatz die Priorität im Statut der Menschen mit Behinderungen gegenüber der im Gesetz über öffentliche Aufzeichnungen (Gesetz 6.015/73) beschriebenen Priorität zu diskutieren. Als Leitfrage: $\mathrm{Ob}$ der Mensch mit Behinderung im Hinblick auf den Menschen mit Behinderung Privilegien gegenüber dem anderen genießt, bei der Weihe der Registralpriorität?

Das Prioritätsprinzip im Immobilienrecht ist die Tatsache, dass es immer Vorrang haben wird, die Eintragungsverfahren durchzuführen, die der Titel zuerst vor dem Grundbuchamt vorgelegt hat. Gemäß Art. 186 des Gesetzes über öffentliche Aufzeichnungen (Gesetz 6.015/73) "bestimmt die Bestellnummer die Priorität des Titels, und dies ist die Präferenz der königlichen Rechte, auch wenn sie von

$\mathrm{RC}: 97618$

Verfügbar in: https://www.nucleodoconhecimento.com.br/gesetz/prioritaetsdienst 
derselben Person mehr als einen Titel gleichzeitig vorgelegt werden", so dass die Priorität für denjenigen bestimmt wird, der den Titel zuerst beim Grundbuchamt einreicht. Und im Protokoll des Notaramtes "nehmen alle Titel die Anzahl der Bestellungen an, die mit innen aufgrund der strengen Reihenfolge ihrer Präsentation konkurrieren" (Art. 182).

In den letzten Jahrhunderten wurde das brasilianische Registrierungssystem ständig durch Gesetze geändert, die darauf abzielt, die Entwicklung der Gesellschaft als Ganzes zu überwachen und die Sicherheit, Publizität und Effektivität von Immobilientransaktionen sowohl für diejenigen, die daran teilnehmen, als auch für diejenigen, die daran interessiert sind, zu ermöglichen. Laut Lima (2011) wirken sowohl notarielle als auch Registrierungstätigkeiten als Mittel der sozialen Befriedung und gewährleisten die Publizität, Authentizität, Sicherheit und Wirksamkeit von Rechtsakten auf präventive Weise.

Es ist wichtig hervorzuheben, dass Notar- und Aufzeichnungstätigkeiten zwar verbunden sind, diese jedoch nicht verwechselt werden sollten. Benício (2005) erläutert, dass dieser Unterschied sichtbar ist, wenn man über die Funktion des Registrars und Notars (Notar) spricht, wobei erstere mit dem Ziel handelt, die Handlungen zu legitimieren und zu bewerben, während die zweite die Beratung in ihren Funktionen mit anschließender Legitimation, Authentizität und Formalisierung von Rechtsakten umfasst. Der Autor unterscheidet immer noch die beiden Aktivitäten und stellt fest, dass der Notar als seine vorherrschende Funktion die dynamische ${ }^{[4]}$ Sicherheit hat, während der Registrar die statische ${ }^{[5]}$ Sicherheit hat, kurz gesagt, während der Notar versucht, das actum (Akt) zu schützen, der Registrar ist auf die Veröffentlichung des dictums beschränkt (gesagt).

Die nach notariellem Recht durchgeführten Tätigkeiten und Registrierungsscans sollten in ihrer sozialen Relevanz gesehen werden, wie Ceneviva (2008) betont, ihre Bedeutung ist auf den Umfang der Handlungen zurückzuführen, die von der Geburt bis zum Tod die Aufzeichnung und Publizität aller von Bürgern während ihres Lebens

RC: 97618

Verfügbar in: https://www.nucleodoconhecimento.com.br/gesetz/prioritaetsdienst 
vorgenommenen Rechtsakte wie Heirat, Scheidung, Teilen, Vorräte, Kauf- und Verkaufsverträge, unter anderem, regularisieren alle durchgeführten Aktionen. Somit handelt es sich um Aktivitäten, die in direktem Zusammenhang mit dem täglichen Leben von Individuen stehen.

Die gesellschaftliche Relevanz dieser Studie wird hervorgehoben, wenn man bedenkt, dass sie eine Minderheit der Bevölkerung betrifft, die bei der Erfüllung ihrer Rechte Aufmerksamkeit benötigt und mögliche Konflikte mit dem Prioritätsprinzip bei der Registrierung von Immobilien sowie die berufliche und akademische Relevanz besser klären kann, da viele immer noch die Kontroversen sind, die sich um das Thema drehen. Da nur wenige Studien zu dem betreffenden Problem durchgeführt und veröffentlicht wurden, bleibt diese Forschung daher gerechtfertigt.

In Bezug auf die verwendete Methodik wird auf der Grundlage von Maria Cecilia de Souza Minayo (2007) hervorgehoben, dass diese Studie in Bezug auf die Zwecke als beschreibend und erklärend eingestuft wird und, was die Mittel betrifft, als bibliographisch eingestuft wird, wobei öffentlich zugängliche Materialien wie Bücher, Artikel, Zeitschriften und Berichte zu diesem Thema verwendet werden. Die Forschung wird auch als rein qualitativer Ansatz charakterisiert. Es wurde als Grundlage genommen, was andere Autoren schrieben, in der Lage zu sein, verschiedene Standpunkte zu diesem Thema zu verstehen und die Studie auf die beste Weise zu lenken, um die Forschung zuverlässig zu machen.

In diesem Zusammenhang zielt die vorliegende Studie, wie bereits erwähnt, darauf ab, den Konflikt zwischen der vorrangigen Pflege des Status von Menschen mit Behinderungen und dem Prinzip der vorrangigen Registrierung von Immobilien zu analysieren.

\section{MENSCHEN MIT BEHINDERUNG IM HISTORISCHEN KONTEXT}

Seit Anbeginn der Zeit gibt es Aufzeichnungen von Menschen mit besonderen Bedürfnissen. Die kulturelle Idee von Behinderung, Impotenz und Abhängigkeit von

$\mathrm{RC}: 97618$

Verfügbar in: https://www.nucleodoconhecimento.com.br/gesetz/prioritaetsdienst 
Menschen mit motorischen Schwierigkeiten durchqueren Kulturen, Gesellschaften, soziokulturelle Gruppen, Religionen und soziale Praktiken. Das Brechen der Erwartung von Eltern mit der Geburt von Kindern, die von einer Art motorischem Problem betroffen sind, der Erwerb einiger Folgen von Krankheiten oder Unfällen, sind sicherlich schwierige Ereignisse und wurden in vielen Kulturen auf atypische Weise erlebt (GUGEL, 2011).

Laut Débora Fazolin Koyama (2017) waren Menschen mit körperlichen Behinderungen immer mit verschiedenen Situationen der Ausgrenzung und Segregation vor der Gesellschaft konfrontiert, in der Zeit des antiken Griechenlands wurde dies besonders vom Stadtstaat Sparta dargestellt, da Kinder, die mit einer Art Behinderung geboren wurden, für diese Gesellschaft als nutzlos angesehen und in einen Abgrund geworfen wurden.

Débora Fazolin Koyama (2017) sagt, dass Menschen mit Behinderungen im Laufe der Geschichte immer einen großen Kampf um das Leben und die Eroberung ihres Platzes in der Gesellschaft geführt haben, da die von der Gesellschaft auferlegte Kultur die Menschen immer marginalisiert und mit diesen Menschen ausschließend gehandelt hat.

Menschen mit Behinderungen begannen, einige ihrer Rechte erst in der Allgemeinen Erklärung der Menschenrechte anerkannt zu bekommen, und von da an wurde ein Kick für das gegeben, was die Standardisierung grundlegender Prinzipien für diese Menschen werden sollte, von diesem Moment an entstand: das Prinzip der Würde der menschlichen Person, das Prinzip der Gleichheit, neben anderen Schutznormen. (KOYAMA, 2017)

Das alte Ägypten war laut Clemente (2015, S. 34) als "Land der Blinden" bekannt, weil seine Menschen ständig von Augeninfektionen betroffen waren, die zur Erblindung führten. Auf der anderen Seite gibt es historische Aufzeichnungen, dass an Orten des antiken Griechenlands und Sparta Kinder mit Missbildungen in den Wäldern ausgesetzt oder aus Schluchten geworfen wurden.

RC: 97618

Verfügbar in: https://www.nucleodoconhecimento.com.br/gesetz/prioritaetsdienst 
Die römischen Gesetze der Antike schützten Menschen mit Behinderungen nicht, im Gegenteil, legitimierten Eltern, ihre Kinder durch die Praxis des Ertrinkens zu töten, die in Körben am Tiber oder an anderen heiligen Orten zurückgelassen wurden, von denen einige freigelegt und als Unterhaltung in Zirkussen verwendet wurden. Studien von Lourenzetto (2006) bestätigen, dass es in den ersten vier Jahrhunderten der christlichen Ära keinen signifikanten Ausdruck gab, der die Eingliederung von Menschen mit Behinderungen als Subjekte der Würde und rechte begünstigte.

In der Antike gibt es nur wenige Aufzeichnungen über die Beziehung der Gesellschaft zu Behinderten im Alltag. Durch Bibelstellen kann die damals bestehende Diskriminierung von Menschen mit Behinderung wahrgenommen werden, weil sie immer als Bettler erwähnt oder von der Gemeinschaft abgelehnt werden, das heißt, sie lebten am Rande des sozialen und gemeinschaftlichen Zusammenlebens. Viele glaubten, dass diese Menschen von den Göttern bestraft wurden (Lourenzetto, 2006, S. 3).

Im Mittelalter galt Behinderung als metaphysisches Phänomen, bestimmt durch dämonische Besessenheit oder als Strafe Gottes. Solche Menschen wurden als "Besitzer eines Bösen aufgrund des Paktes mit dem Teufel" angesehen, eine Rechtfertigung, die verwendet wurde, um den extremen Gebrauch des Lagerfeuers als Strafe sozial zu legitimieren (MAINIERI; ROSA, 2012).

Es wurde angenommen, dass eine solche Praxis es ermöglichte, den vermeintlichen Feind, der besiegt werden musste, zu demütigen und zu gewinnen. Für Maria Aparecida Gugel (2011) wurden die überlebenden Kinder nicht nur Menschen mit Behinderungen besondere Zauberkräfte zu geben, sondern fast immer von ihren Familien getrennt und verspottet.

Marcella Lourenzetto (2006) verweist in päpstlichen Dokumenten darauf, dass er im Falle einer Person mit geistiger Behinderung, die vor seiner Vernunft floh, als teuflisches Wesen galt, das verfolgt, gefoltert und ausgerottet wurde. Im 15. Jahrhundert wurden solche Individuen noch nicht als Menschen wahrgenommen.

RC: 97618

Verfügbar in: https://www.nucleodoconhecimento.com.br/gesetz/prioritaetsdienst 
In diesen Beispielen wird deutlich, wie Segregation und Fatalismus Menschen mit Blindheitsproblemen und alle anderen betrafen, die vor etablierten Standards als normal wegliefen (BIANCHETTI; FREIRE, 2007). Die Amputationsstrafe wurde auch als Kontrolle und Bestrafung von Verrätern in den römischen Verfassungen von Kaiser Leo III. verwendet, ein Prozess, der im Römischen Reich und im Osten vorherrschte.

Die in der Zeit der Inquisition nob eingeführte Argumentation übernahm die Praxis, Menschen zu verbrennen, die einen Unterschied in ihren Körper brachten, der als nicht normal galt, oder die unterschiedliche Vorstellungen vom Status quo präsentierten oder sich in einer Weise verhielten, die als ungeeignet angesehen wurde. Aus diesem Grund gibt es in den Aussagen der Inquisition und in den Begründungen der Kirche keine Aussagen, dass sie Menschen verbrannt hat. Wie Lucídio Bianchetti und Ida Maria Freire (2007, S. 33) feststellen, hat die Kirche eine solche Aktion als "[...] Reinigung durch Flammen" ausgesprochen. Eine andere Erklärung für die Existenz von blinden, stummen, gelähmten, wahnsinnigen und aussätzigen Menschen war, dass diese wie möglich konzipiert wurden:

[...] Gottes Werkzeuge, um Männer und Frauen vor angemessenem Verhalten zu warnen oder inm die Möglichkeit zu geben, Nächstenliebe zu tun. So bot das Unglück einiger mittel zur Erlösung für andere. (BIANCHETTI; FREIRE, 2007, S. 33).

Laut Maria Aparecida Gugel (2011) wird der Prozess der Menschen mit Behinderungen als Menschen der Würde und des Wertes anerkannt, die mit den ersten Krankenhäusern der Nächstenliebe kamen. Für den Autor gründete König Luís IX. zwischen 1214 und 1270 das erste Krankenhaus für Blinde, die Opfer der Kreuzzüge wurden. Die Konstruktion neuer Vorstellungen von Behinderung war historisch erst im sechzehnten Jahrhundert möglich, als technologische und wissenschaftliche Fortschritte die Entstehung unterschiedlicher Vorstellungen in der Medizin ermöglichten.

$\mathrm{RC}: 97618$

Verfügbar in: https://www.nucleodoconhecimento.com.br/gesetz/prioritaetsdienst 
Erst im 16. Jahrhundert, mit wissenschaftlichen Fortschritten auf dem Gebiet der Medizin, erschienen die ersten dissonierten Aussagen über die Behandlung von Behinderten (GUGEL, 2011).

Die meisten Männer und Frauen waren darauf beschränkt, ihren Alltag auf miserable Weise zu leben und mit der Produktion für den Lebensunterhalt zu tun. Mit der allmählichen Vorherrschaft der marktorientierten Produktion erhält die Möglichkeit der Akkumulation, die Entwicklung einer Wissenschaft und neuer Technologien eine relative Dominanz über die Natur. Mit diesem Prozess wurden materielle, soziale und kulturelle Bedingungen für die Konstruktion emanzipatorischer Prozesse geschaffen, die das Durchzugsland der Sphäre der Notwendigkeit für Freiheit ermöglichen.

Erst im 16. Jahrhundert war ein anderer historischer Prozess möglich, der sich von der bisherigen historischen Situation unterschied. Mit den Fortschritten in der Medizin, die im 17. Jahrhundert auftraten, wurde körperliche Behinderung als Gegenstand des Studiums der medizinischen Praxis verstanden. Der Prozess der Medizinischenlsierung von Mängeln, der in der Kritik der historisch gegenwärtigen Visionen impliziert wird, dass in jedem Menschen über einen morbiden Zustand herrschte, die Anwesenheit böser Dämonen, die Gesundheit und Krankheit beeinflussen (BIANCHETTI; FREIRE, 2007).

Die metaphysischen Erklärungen der religiösen Sphäre werden mit dem positivistischen wissenschaftlichen Paradigma konfrontiert, so dass die theologische Sicht auf Differenz an Kraft verliert, aber die theoretischen Grundlagen für eine organisch-organische Interpretation beeinflusst. Im 17. Jahrhundert wurde ein großer Kreuzgang all derer entfesselt, die nicht an der entstehenden Universellen Vernunft teilnehmen. Derjenige, der die Abwesenheit der Vernunft in irgendeiner ihrer Formen als logische, politische, moralische Vernunft zeigte, wurde geschlossen (BIANCHETTI; FREIRE, 2007).

Erst ab Mitte des 19. Jahrhunderts entstanden die ersten Einrichtungen zur Betreuung von Menschen mit körperlichen Behinderungen. Lange Zeit galten solche

$\mathrm{RC}: 97618$

Verfügbar in: https://www.nucleodoconhecimento.com.br/gesetz/prioritaetsdienst 
Menschen nur als besonders pflegebedürttige Patienten und diese als Menschen mit geringem Potenzial und mit zahlreichen Einschränkungen. In Brasilien wurden im gleichen Zeitraum das Kaiserliche Institut für blinde Jungen und das Kaiserliche Institut für Taubstumme im Auftrag von D. Pedro II. gegründet, das im Auftrag von D. Pedro II. und des Kaiserlichen Instituts der Taubstummen gegründet wurde, eine Tatsache, die als eine der ersten brasilianischen Einstellungen für die Integration von Behinderten in die Gesellschaft gilt (SOUSA, 2012).

Die Schaffung von Klostergeräten erzeugte jedoch auch Prozesse der Segregation des sozialen Umfelds, was dazu führte, dass dies die Konstruktion dessen war, was Erving Goffman (2008, S.13) als Stigma definiert, "[...] eine besondere Art der Beziehung zwischen Attribut und Stereotyp", die im Kontext menschlicher Beziehungen und nicht substantiell verstanden wird. "Ein Attribut, das jemanden stigmatisiert, kann die Normalität anderer bestätigen."

Das Thema Behinderung nimmt aufgrund der Beobachtung des in den 1970er Jahren genickten Paradigmenwechsels des letzten Jahrhunderts einen weiteren Schwerpunkt ein, integrative Maßnahmen werden befürwortet, um die Anwesenheit / Eingliederung von Menschen mit Behinderungen in die gleichen sozialen Sphären wie die anderen zu fördern. Diese Möglichkeit erscheint jedoch in ihrer realen Funktionalität als etwas Utopisches, denn wie Erving Goffman erklärt (2008, S. 134):

Die besondere Situation des Stigmatisierten ist, dass die Gesellschaft inm sagt, dass er ein Mitglied der breiteren Gruppe ist, was bedeutet, dass er ein normaler Mensch ist, aber auch, dass er bis zu einem gewissen Grad "anders" ist und dass es absurd wäre, diesen Unterschied zu leugnen. Der Unterschied selbst leitet sich von der Gesellschaft ab, denn im Allgemeinen muss ein Unterschied, bevor er wichtig ist, von der Gesellschaft als Ganzes kollektiv konzeptualisiert werden.

So nimmt die Eingliederung dieser Öffentlichkeit in die verschiedenen sozialen Bereiche jedes Jahr zu, obwohl physische Einrichtungen, die Bereitstellung von Material und die Ausbildung von Fachkräften zur Betreuung von Menschen mit besonderen Bedürfnissen immer noch prekär sind, wobei diese Studie auf die

RC: 97618

Verfügbar in: https://www.nucleodoconhecimento.com.br/gesetz/prioritaetsdienst 
Rechte dieser Bürger konzentriert wird, insbesondere integration, inklusion und gleichheit, basierend auf dem Grundprinzip der Würde der menschlichen Person.

\section{MENSCHENRECHTE MIT SCHWERPUNKT AUF MENSCHEN MIT BEHINDERUNGEN}

Menschenrechte mit Schwerpunkt auf Menschen mit Behinderungen müssen neben der Würde der menschlichen Person und der Gleichheit das Recht auf Mobilität und die Stadt sowie Bildung und Gesundheit hervorheben, da sie entscheidende Faktoren sind, um die soziale Inklusion dieser Menschen zu gewährleisten. Während des gesamten Evolutionsprozesses der menschlichen Zivilisation gab es die Suche nach Rechten, die von der Fortbewegung bis zur Zirkulation in öffentlichen und privaten Umgebungen unabhängig voneinander reichten.

Historisch gesehen wurden die Menschen missachtet und völlig vom sozialen Leben ausgeschlossen, insbesondere vom sozialen Kontext, und dies basierte auf den Vorstellungen, die sie über den Menschen und die Gesellschaft hatten, die Individuen eingeimpf wurden, die ein mentales Modell bildeten, das sich darin niederlässt und nicht mehr verschwindet. Es transformiert sich einfach (LOPES et al., 2009, S. 29).

Die Perspektive der historisch erreichten Bürgerrechte hat die Idee von Integration, Inklusion, Gleichheit als eine Möglichkeit zur Bewältigung der Prozesse der sozialen Ausgrenzung, Diskriminierung und Stigmatisierung gebracht. Dieser Prozess ist grundlegend, wie Bürgersteige, die das Recht auf Kommen und Gehen jedes Bürgers in der Stadt, in der er lebt, garantieren.

In Brasilien beinhaltete dieser Prozess die Anerkennung des Rechts auf Barrierefreiheit. In der Bundesverfassung, Kapitel VII, sieht Art. 227 die «Erleichterung des Zugangs zu kollektiven Gütern und Dienstleistungen unter Beseitigung architektonischer Vorurteile und Hindernisse» vor und enthält damit Regeln, die den Bau von Orten, öffentlichen Gebäuden und öffentlichen Verkehrsmitteln garantieren. (NOGUEIRA, 2010, S. 51). So haben die Vereinten

RC: 97618

Verfügbar in: https://www.nucleodoconhecimento.com.br/gesetz/prioritaetsdienst 
Nationen - UNO 1981 in einer Konvention das Internationale Jahr der Menschen mit Behinderungen anerkannt. 1989 wurden diese Rechte durch das Gesetz Nr. 7.853, deren Zweck es ist, "den gerichtlichen Schutz kollektiver oder diffuser Interessen dieser Personen zu etablieren, diszipliniert die Leistung der Staatsanwaltschaft, definiert Straftaten und sieht andere Maßnahmen vor" (BRASIL, 1989). Das gleiche Gesetz bestimmt die Einhaltung der Durchsetzung der Vorschriften durch die Gemeinden, um die Funktionalität von Gebäuden und öffentlichen Straßen für Behinderte zu fördern.

Schließlich ist das Bundesgesetz Nr. 10.098 vom 19. Dezember 2000[6], später geregelt durch dekret Nr. 5.296 vom 2. Dezember 2004[7], Standardisierung des Themas Barrierefreiheit als Anpassungsziel und Zugangsinstitution. Bis heute wurde dieses Gesetz jedoch nicht umgesetzt, da Elemente wie der Zugang zu öffentlichen Gebäuden, die Dauerhaftigkeit und die Nutzung dieser Gebäude, wie Bildung und Gesundheit, nicht vollständig berücksichtigt wurden. Bürgersteige sind das Porträt der ausschließenden Gesellschaft, die es notwendig macht, Notfallprobleme wie Löcher in den Straßen, Autos auf Gehwegen, die die Mobilität unterbrechen, Standardisierung von Rampen, Handläufen, Gehwegen, öffentlichen Toiletten und speziellen Beschilderungen zu lösen, damit Zugänglichkeitsprobleme minimiert werden können (COELHO, 2010).

In diesem Sinne ist das Recht auf Barrierefreiheit ein unverzichtbarer Faktor nicht nur für diejenigen, die eine Art Behinderung oder eingeschränkte Mobilität haben, sondern für die Gesellschaft als Ganzes, denn auf der Grundlage des Gleichheitsprinzips ist jeder vor dem Gesetz gleich und genießt die gleichen Rechte auf Kommen und Gehen, Bildung, Freizeit, Wohnen, Tourismus und Kultur. Gemäß der Erklärung der Rechte von Menschen mit Behinderungen, die 1975 auf einer Generalversammlung der Vereinten Nationen (UN) verabschiedet wurde, heißt es unter anderem: "Behinderte Menschen haben Anspruch auf Maßnahmen, die sie befähigen sollen, so selbstbewusst wie möglich zu werden."

$\mathrm{RC}: 97618$

Verfügbar in: https://www.nucleodoconhecimento.com.br/gesetz/prioritaetsdienst 
Die Weltcharta des Rechts auf Stadt, die auf dem Sozialforum Amerikas in Quito, dem World Urban Forum in Barcelona im Jahr 2004 und dem Fünften Weltsozialforum von Porto Alegre im Jahr 2005 verabschiedet wurde, legt Verpflichtungen zwischen Regierungen und der organisierten Zivilgesellschaft fest, Städte auf der Grundlage der Prinzipien der Solidarität, Freiheit, Gleichheit, sozialen Gerechtigkeit und Würde zu fördern.

Das Recht auf Stadt wird in der Charta definiert als: "Der gerechte Genuss von Städten innerhalb der Prinzipien der Nachhaltigkeit, Demokratie und sozialen Gerechtigkeit [...] ist von allen international anerkannten Rechten abhängig". Die Stadt wird in diesem Dokument als "kulturell reicher und diversifizierter kollektiver Raum, der allen Bürgern gehört" konzipiert (CARTA MUNDIAL DO DIREITO À CIDADE, 2004, S. 2). Was den besonderen Schutz schutzbedürftiger Gruppen und Personen betrifft, so heißt es in der Stadtcharta:

Städte müssen durch eine Politik der positiven Bestätigung für schutzbedürftige Gruppen politische, wirtschaftliche und soziale Hindernisse überwinden, die die Freiheit, Gleichheit und Gleichheit der Bürger einschränken und die volle Entwicklung der menschlichen Person und die effektive Teilnahme an der politischen, wirtschaftlichen, kulturellen und sozialen Organisation der Stadt verhindern (CARTA MUNDIAL DO DIREITO À CIDADE, 2004, S. 3).

In Bezug auf die städtische Mobilität heißt es in Artikel 13:

1. Städte garantieren das Recht auf Mobilität und Bewegung in der Stadt durch ein System und öffentliche Verkehrsmittel, die für alle Menschen nach einem Plan für Stadt- und Fernreisen zugänglich sind, und auf der Grundlage der geeigneten Verkehrsmittel die verschiedenen sozialen Bedürfnisse (Geschlecht, Alter, Behinderung) und Umwelt, mit Preisen, die dem Einkommen der Bürger entsprechen. Die Verwendung von nicht kontaminierenden Fahrzeugen wird gefördert und

RC: 97618

Verfügbar in: https://www.nucleodoconhecimento.com.br/gesetz/prioritaetsdienst 
Fußgängerzonen werden zu bestimmten Tageszeiten dauerhaft reserviert.

2. Die Städte werden die Beseitigung architektonischer Barrieren für die Implementierung der notwendigen Ausrüstung für das Mobilitäts- und Verkehrssystem und die Anpassung aller öffentlichen oder öffentlichen Gebäude und Arbeitsplätze fördern, um die Zugänglichkeit von Menschen mit besonderen Bedürfnissen zu gewährleisten (CARTA MUNDIAL DO DIREITO À CIDADE, 2004, S. 3).

Es ist erwähnenswert, dass, wenn es um Zugänglichkeit geht, es nicht auf den physischen Raum beschränkt ist, in dem es installiert ist, es ist die Stadt als Ganzes, die den politischen, sozialen, wirtschaftlichen und intellektuellen Prozess der Gesellschaft darstellt.

Im Bereich Bildung und Gesundheit wird betont, dass die Schulinklusion als ein Recht des Bürgers konfiguriert ist, das universell garantiert, wobei als Meilenstein dieses Prozesses die Salamanca-Erklärung hervorgehoben wird, die eine inklusive Bildung unter Einhaltung der Konventionen und Menschenrechte betont, die sich an Kinder und Jugendliche richtet und hier die wichtigsten als Verdienst der Grundkenntnisse des Themas darstellt.Das Dokument wurde nach einer Weltkonferenz über Sonderpädagogik in Salamanca im Jahr 1994 benannt, die darauf abzielte, die politische Reform nach Inklusion umzustrukturieren, und erklärte:

"Jedes Kind hat ein Grundrecht auf Bildung und sollte die Möglichkeit
erhalten, ein angemessenes Lernniveau zu erreichen und
aufrechtzuerhalten, jedes Kind hat Merkmale, Interessen, Fähigkeiten
und Lernbedürfnisse, die einzigartig sind, Bildungssysteme sollten
festgelegt werden und Bildungsprogramme sollten durchgeführt
werden, um der großen Vielfalt dieser Merkmale und Bedürfnisse
Rechnung zu tragen. Personen mit sonderpädagogischem
Förderbedarf sollten Zugang zur Regelschule haben, die in der Lage
sein sollte, sie im Rahmen einer kindzentrierten Pädagogik zu
unterstützen, die in der Lage ist, diesen Bedürfnissen gerecht zu
werden, reguläre Schulen, die über eine solche inklusive Beratung
verfügen, sind das wirksamste Mittel, um diskriminierende RC: 97618

Verfügbar in: https://www.nucleodoconhecimento.com.br/gesetz/prioritaetsdienst 
Einstellungen zu bekämpfen, indem sie einladende Gemeinschaften schaffen, eine integrative Gesellschaft aufbauen und Bildung für alle erreichen; Darüber hinaus bieten solche Schulen den meisten Kindern eine effektive Bildung und verbessern die Effizienz und letztendlich die Kosten für die Wirksamkeit des gesamten Bildungssystems (...)" So zitiert das Dokument (ONU, 2010, S. 78).

Diese Proklamation verpflichtet uns, Kindern und Jugendlichen spezifische Programme und maßgeschneiderte Evaluierungen anzubieten und Möglichkeiten zu bieten, um die volle Entwicklung und Entwicklung des Potenzials jedes Einzelnen zu gewährleisten. Dazu sollten die Anpassungen des Lehrplans berücksichtigt werden und die Garantie des Gesetzes in Betracht gezogen werden.

Dies ist ein internationales Abkommen über die Rechte von Kindern und Jugendlichen. In Übereinstimmung mit den in der Charta der Vereinten Nationen proklamierten Grundsätzen ist die Anerkennung der allen Mitgliedern der Menschheitsfamilie innewohnenden Würde und der gleichen und unveräußerlichen Rechte die Grundlage für Freiheit, Gerechtigkeit und Frieden in der Welt, wobei die Völker der Vereinten Nationen in ihrer Charta ihr Vertrauen in die grundlegenden Menschenrechte, die Würde und den Wert der menschlichen Person bekräftigt und entschieden haben, Förderung des sozialen Fortschritts und eines besseren Lebensstandards in größerer Freiheit.

Die Nationale Politik zur Sonderpädagogik (PNEE) aus der Perspektive inklusiver Bildung (PEI) (2008) hebt die Notwendigkeit hervor, der Segregation von Schülern im schulischen Umfeld entgegenzutreten, und schlägt eine Sonderpädagogik vor, die sich komplementär entwickelt und das einzigartige Bildungssystem bekräftigt.

In diesem Zusammenhang sind die Menschenrechte universell und kein Bürger kann aus irgendeinem Grund aufgrund seiner körperlichen, geistigen Bedingungen oder aufgrund seiner Hautfarbe, Rasse, Sitte oder sozialen Klasse ausgeschlossen werden, daher liegt es an den Behörden, öffentliche Richtlinien zu entwickeln, um sich gleichermaßen um diese Personen zu kümmern.

RC: 97618

Verfügbar in: https://www.nucleodoconhecimento.com.br/gesetz/prioritaetsdienst 
In der Erkenntnis, dass Menschen mit Behinderungen unter anderem die Bevorzugte, schnelle und individuelle Betreuung bevorzugen, erkennen sie den Genuss der in der Bundesverfassung von 1988 verankerten Grundrechte. Es sollte jedoch klargestellt werden, dass dieses Recht, da es sich um ein sehr persönliches Recht handelt, zugunsten der Person mit Behinderungen oder von Personen, die sich in einem Zustand der Schutzbedürftigkeit befinden, gemäß dem Bundesgesetz 10.048 vom 8. November 2000[8] ausgeübt werden sollte, das den Priorität der Pflege den darin festgelegten Personen weihte: Menschen mit Behinderungen, schwangere Frauen, stillende Frauen, Menschen, die von Kindern in ihrem Leben begleitet werden, zusätzlich zu älteren Menschen.

Es ist auch belegt, dass der persönliche Zustand der Partei nicht der Haupthöhepunkt des Statuts der Person mit Behinderungen ist, was angestrebt wird, ist die bevorzugte, sofortige und individuelle Betreuung des schutzbedürftigen Teils, in unserer Studie, der Person mit Behinderungen in Notaren.

\section{STATUS VON MENSCHEN MIT BEHINDERUNGEN UND DAS PRIORITÄTSPRINZIP DER REGISTRIERUNG VON IMMOBILIEN}

Im Zusammenhang mit Notar- und Registrierungsdiensten ist es neben der Einhaltung der Grundprinzipien der öffentlichen Verwaltung erforderlich, dass Notawills und Registrare auch Grundsätze erfüllen, die als spezifisch für diese Tätigkeiten gelten. Es ist wichtig zu verstehen, dass die Prinzipien formbar sind, da sie sich an die historischen und sozialen Umstände anpassen, die sie durchmachen.

Die Grundsätze, die auf die Registrierungsdienste angewendet werden, umfassen die Maßnahmen, die während des gesamten Registrierungsprozesses, von der Registrierung bis zur Rechtshilfe, im Zusammenhang mit dem Immobilienrecht durchzuführen sind. Das Prinzip der Registrierung ist laut Carvalho (2011) der Beginn des Prozesses, der für die Bildung, Übertragung, Änderung oder Das Erlöschen der Rechte in ris verantwortlich ist. Es handelt sich also um die

RC: 97618

Verfügbar in: https://www.nucleodoconhecimento.com.br/gesetz/prioritaetsdienst 
Aufzeichnung von Handlungen, die eine Registrierungshandlung ändern oder löschen.

Das bereits erwähnte Prinzip der Werbung als Grundprinzip der öffentlichen Verwaltung wird weiterhin in Betracht gezogen, und Registrare sollten die in ihren Notartorien gemachten Aufzeichnungen veröffentlichen. Nach Ceneviva (2008) zielt die Werbung als Prinzip der Registrierungstätigkeit darauf ab, ihre dreifache Funktion zu erfüllen, nämlich die Registrierung an Dritte weiterzugeben, über Vermögenswerte und Rechte von Personen zu informieren, die Vorteile aus der Registrierung erhalten, ihr Recht auf Privatsphäre und Intimität zu opfern und Handlungen für statistische Zwecke zu fördern, nationales Interesse oder öffentliche Aufsicht.

Der Grundsatz der Wahrheitsvermutung, der auf die registrierten Dienste angewandt wird, ist auch ein Ergebnis des öffentlichen Glaubens, das ihnen zusammen mit der Übertragung der Funktion gewährt wird. Vasconcelos und Cruz (2000) erklären, dass sich das öffentliche Vertrauen auf alle Handlungen erstrecken muss, die im Rahmen von Registrierungsdiensten durchgeführt werden, und positiv auf das Bestehen echter Rechte reagieren muss. So wird immer davon ausgegangen, dass alles, was im Grundbuch eingetragen ist, wahr ist, bis das Gegenteil bewiesen ist.

Das Prioritätsprinzip wiederum befasst sich mit der Frage der Reihenfolge der Eintragungen bei der Registrierung von Immobilien, und der Registrar sollte die chronologische Reihenfolge der Präsentation der Titel gemäß Art. 186 des Gesetzes Nr. 6,015/1973 berücksichtigen. Antunes (2005) weist darauf hin, dass es die chronologische Reihenfolge ist, die die Priorität des Titels und die Präferenz des wirklichen Rechts bestimmen sollte.

Das Prinzip der Spezialisierung oder Bestimmung von Aufzeichnungen führt dazu, dass die Registrierung so erfolgen muss, dass die Beschreibung der Immobilie korrekt ist und mit ihren besonderen Merkmalen wie Registrierung, Bestellnummer, Datum, Identifizierung der Immobilie, Konfrontationen, Standort und Bereich. Darüber hinaus ist es auch notwendig, Daten seines Eigentümers wie Name, Wohnsitz,

$\mathrm{RC}: 97618$

Verfügbar in: https://www.nucleodoconhecimento.com.br/gesetz/prioritaetsdienst 
Nationalität, Familienstand, Beruf, allgemeine Registernummer (RG) und im Falle einer juristischen Person in die Sozialgesellschaft und die Nummer des Nationalen Registers der juristischen Personen (CNPJ) aufzunehmen. Die korrekte Beschreibung dieser Daten ist unerlässlich, um Rechtssicherheit für die durchgeführten Handlungen zu schaffen (ANTUNES, 2005).

Die Fortsetzung der Beschreibung der Grundsätze der registrierten Dienste hat den Grundsatz der Qualifikation, Rechtmäßigkeit oder Legitimität, dass der Registrar laut Antunes (2005) den vorgelegten Titel unter Berücksichtigung der Form, Gültigkeit und Einhaltung des Gesetzes prüfen muss. Galiani (1995) erwähnt auch, dass der Registrar selbst zur Prüfung des inm verliehenen Titels von der Gesetzgebung Gebrauch machen muss, nicht in der Lage sein muss, die gesetzlich festgelegten Grenzen zu überschreiten, und nur die formalen Aspekte davon analysieren sollte.

Das Prinzip der Kontinuität ist auch Teil der Liste der Prinzipien, die auf Registrierungsdienste angewendet werden, und wird von Antunes (2005) als eine der Grundlagen dieser Dienste hervorgehoben. Zur Anwendung dieses Prinzips erläutert Balbino Filho (2001), dass es die Verpflichtung zur Kontinuität der Registrierung mit sich bringt und die Verbindung zwischen den verschiedenen Unternehmen aufrechterhält, die die rechtliche Situation geändert haben. Daher müssen alle Änderungen, die im Titel auftreten, im selben Dokument aufgezeichnet werden, wobei die vorherigen Informationen erhalten bleiben.

Schließlich gibt es den Grundsatz der Instanz oder des Rechtszugs, der sich mit den Maßnahmen befasst, die der Registrar auf der Grundlage des Antrags der Parteien ergreift. Nach Balbino Filho (2001) betrifft der Grundsatz der Instanz oder des Rechtszugs die Aufforderung zu jeder registralen Handlung und muss einfach, in besonderer Weise unabhängig und kann ausdrücklich oder stillschweigend sein. Ausgedrückt, wenn es von den Parteien klar zum Ausdruck gebracht wird, und stillschweigend, wenn der Registrar den Willen der Parteien identifizieren kann, indem er ihre Erfahrungen erzählt.

$\mathrm{RC}: 97618$

Verfügbar in: https://www.nucleodoconhecimento.com.br/gesetz/prioritaetsdienst 
In Anbetracht des Vorstehenden müssen die Registrierungsdienste in Übereinstimmung mit den genannten Grundsätzen erbracht werden, und es darf nicht vergessen werden, dass die Grundprinzipien der öffentlichen Verwaltung bei allen Tätigkeiten berücksichtigt werden sollten, die von Registraren im Rahmen ihrer Aufgaben ausgeübt werden.

Diskussionen über den Grundsatz des Vorrangs bei der Eintragung von Eigentum wurden so kontrovers geführt, dass es möglich ist, entschiedene Fälle zu identifizieren, in denen Konflikte mit den Rechten bestimmter Zielgruppen berücksichtigt werden. Lago (2016) zitiert die Gewährung der Priorität bei der Unterstützung von Rechtsanwälten und die Gewährung der vorrangigen Unterstützung für ältere Menschen, beide aus dem Register von São Paulo - SP. Im ersten Fall wurde sie aufgrund der spezifischen gesetzlichen Bestimmung zu Gesetz 8.906/1994 - Statut der Brasilianischen Anwaltskammer verneint und die Rangordnung verletzt, jedoch wurde empfohlen, vorrangige Behandlung in Fällen zu gewähren, in denen Notare und eingetragene Richter, nach vorsichtigen Kriterien, erforderlich.

Im zweiten Fall verwendeten die Richter die Kriterien des Gesetzes 6.015/1973 und des Bürgerlichen Gesetzbuches, wonach die vorgelegte Anordnung strikt befolgt werden sollte. Damit blieb das Verständnis, dass ältere Menschen bei der Bereitstellung von Zertifikaten oder bei der Zustellung von Dokumenten vorrangig behandelt werden, nicht jedoch bei der Eintragung von Titeln, die Priorität generieren könnten (LAGO, 2016).

Daraus lässt sich dieses Verständnis auch bei Menschen mit Behinderungen nehmen, da sein Statut auch das Recht auf vorrangige Pflege vorsieht. Es wurde festgestellt, dass nach Ansicht der eingetragenen Gerichte die spezifische Bestimmung des Gesetzes Nr. 6,015/1973 in Bezug auf das Recht auf vorrangige Betreuung bestimmter Zielgruppen Vorrang hat, da sie sich auf ein materielles Prioritätsrecht bezieht, wobei davon ausgeht, dass die Gewährung einer vorrangigen

RC: 97618

Verfügbar in: https://www.nucleodoconhecimento.com.br/gesetz/prioritaetsdienst 
Betreuung außerhalb der Anforderungen von Art. 186 des Gesetzes Nr. 6,015/1973 eine unbestreitbare Anerkennung außerrechtlicher Gründe darstellen würde. was den Dienst am Recht auf Gleichheit gefährden würde, eine Prämisse auch des Statuts der Person mit Behinderungen.

Das Prinzip der Priorität ist in den Künsten festgelegt. 182 und folgt des Gesetzes Nr. 6,015/73. Es wird zum Beispiel erwähnt, dass seine Auswirkungen auf die Hypothek und die sie umgebenden Handlungen, obwohl sie redundant sind, auch im Bürgerlichen Gesetzbuch, in Art. 1493, in verbis enthalten sind: "Die Aufzeichnungen und Vermerke folgen der Reihenfolge, in der sie angefordert werden, und überprüfen die ihrer aufeinanderfolgenden Nummerierung im Protokoll." Somit gibt es keinen Antrag einer behinderten Person, der ihren Antrag vor denjenigen erfüllt, die ihm in einem Standesamt nachgefolgt sind. In diesem Sinne Afrânio Carvalho (2011):

(...) In einem Wettbewerb der tatsächlichen Rechte an einem Eigentum nehmen sie nicht alle den gleichen Rang ein, sondern graduieren oder qualifizieren sich durch ein Vorrangverhältnis, das auf der chronologischen Reihenfolge seines Erscheinens basiert: prior tempore potior jure. Abhängig von der Zeit, in der sie entstehen, nehmen die Rechte eine Position im Register ein und haben Vorrang vor denen, die danach kommen. (CARVALHO, 2011, S. 216).

Es wird erläutert, dass die Titel, die ein Prioritätsrecht generieren, bei der Veröffentlichung im Buch Nr. 1 - Protokoll gemäß der strengen Reihenfolge der Präsentation, die im Gesetz über öffentliche Aufzeichnungen beschrieben ist, in das Immobilienregister aufgenommen werden müssen. Aus diesem Grund erlaubt es ein Recht auf bevorzugte Pflege aufgrund von Behinderung, Alter und Geschlecht nicht, im Sinne dieser Titel vor anderen zu erhalten, die bereits die Räumlichkeiten des Dienstes betreten haben. Unter Anwendung des Grundsatzes der Eintragungspriorität sollte in erster Linie ein Titel nach der niedrigsten Protokollnummer eingetragen werden, "wodurch die Eintragung der später vorgelegten Personen für den Zeitraum, der mindestens einem Arbeitstag entspricht" (Gesetz 6.015/73, Art. 191). Obwohl der Grundsatz der Priorität für Behinderte nicht angewendet wird, sollten die Titel in beiden Fällen eingetragen werden.

$\mathrm{RC}: 97618$

Verfügbar in: https://www.nucleodoconhecimento.com.br/gesetz/prioritaetsdienst 
Um das Problem zu lösen, schlägt Lago (2016, S. 316) "ein System eines Systems vor, das aus einem Passwort-Provider-Gerät mit bestimmten Eigenschaften und zwei gleichzeitigen Protokollbüchern - einem vorläufigen und einem definitiven - besteht". Es wird jedoch angenommen, dass das Problem darüber hinausgeht und für eine Lösung nicht ausreicht, da es angesichts der Autonomie des Registralsystems und der Absicht, das Statut der Person mit Behinderungen zu erlassen, nicht ausreicht, es wird davon ausgegangen, dass die vorrangige Pflege in diesem Fall nicht stattfinden sollte, da sonst das Recht auf Gleichheit beeinträchtigt würde.

\section{FAZIT}

Die Notar- und Registrierungsdienste wurden in dieser Studie angesprochen, wobei sie in inrer Tätigkeit berücksichtigt wurden, die von der Privatsphäre durch Delegation der öffentlichen Macht ausgeübt wird, wobei sie sich auf das soziale Interesse konzentrieren, daher aufgrund des Gehorsams gegenüber den allgemeinen Grundsätzen der öffentlichen Verwaltung, zusätzlich zu den für diese Aktivitäten spezifischen Grundsätzen, die in jedem Akt beachtet werden müssen.

Neben dem Gehorsam gegenüber bestimmten Grundsätzen dieser Tätigkeiten, die die der Inschrift, der Publizität, der Vermutung der Wahrheit, der Priorität, der Spezialisierung oder Bestimmung der Aufzeichnungen, der Qualifikation, Rechtmäßigkeit oder Legitimität, der Kontinuität und der Instanz oder des Rechtszugs sowie des öffentlichen Glaubens sind, der eine der wichtigsten in der Rechtsprechung genannten ist.

Das Thema wurde aus der Perspektive behandelt, dass, obwohl die Rechte von Menschen mit Behinderungen in der Bundesverfassung garantiert sind, die Realität eine große Distanz zwischen formalen Rechten und ihrer Wirksamkeit offenbart, was bestätigt, dass es im Laufe der Geschichte zahlreiche öffentliche Politiken gab, die sich an Menschen mit Behinderungen richteten, um Barrierefreiheit zu erreichen. Mobilität und Gleichberechtigung, ihre Integration in die Gesellschaft, aber es ist

RC: 97618

Verfügbar in: https://www.nucleodoconhecimento.com.br/gesetz/prioritaetsdienst 
offensichtlich, dass es noch einen bedeutenden Weg gibt, der befolgen werden muss, um ihre Rechte wirksam zu erfüllen.

Innerhalb des Registrierungssystems wurde festgestellt, dass es einen Konflikt zwischen seinen Bestimmungen des Grundsatzes der Registrierungspriorität und dem Statut der Person mit Behinderungen gibt, das eine vorrangige Betreuung dieser Öffentlichkeit vorsieht. Es stellt sich heraus, dass in diesem System materielle Rechte fokussiert sind, daher würde die Berücksichtigung des Statuts der Person mit Behinderungen für die vorrangige Pflege Konsequenzen wie die Möglichkeit haben, das im Land geltende Rechtssystem zu umgehen oder zu betrügen.

So wurde aus ähnlichen Entscheidungen der Register von São Paulo festgestellt, dass die spezifische Bestimmung des Gesetzes Nr. 6,015/1973 in Bezug auf das Recht auf vorrangige Betreuung bestimmter Personen Vorrang hat, da sie sich auf ein materielles Prioritätsrecht bezieht, wobei davon ausgeht, dass die Gewährung einer vorrangigen Pflege außerhalb der Anforderungen von Art. 186 des Gesetzes Nr. 6,015/1973 wäre eine unbestreitbare Anerkennung eines Affronts gegen die wirklichen Rechte der Bürger, der den Dienst am Recht auf Gleichheit, einer Prämisse auch des Statuts der Menschen mit Behinderungen, gefährden würde.

Schließlich, als eine Möglichkeit, die Frage zu beantworten, die der Leitfaden dieses Aufsatzes ist, wird akzeptiert, dass die Garantie des Mechanismus der Registrierungspriorität nicht im Gegensatz zu der allgemein etablierten Fakultät im Statut der Menschen mit Behinderungen steht. Die Regeln hierfür sind nicht den Regeln des Gesetzes 6.015/1973 auferlegt. Sie beschäftigen sich mit solchen Diplomen in verschiedenen Situationen. Mit anderen Worten, es lohnt sich die Schutzregel bis zu der Grenze, dass sie nicht durch eine andere, von der öffentlichen Ordnung, die den Interessierten den betroffenen Parteien den vorrangigen Vorrang und am entfernten Ende die Präferenz und Definition der tatsächlichen Rechte entweiht, ausgeschlossen wird.

RC: 97618

Verfügbar in: https://www.nucleodoconhecimento.com.br/gesetz/prioritaetsdienst 


\section{VERWEISE}

ANTUNES, Luciana Rodrigues. Introdução ao Direito Notarial e Registral. Jus Navigandi, Teresina, ano 9, n. 691, 2005.

BIANCHETTI, Lucídio; FREIRE, Ida Mara (orgs.). Um olhar sobre a diferença: interação, trabalho e cidadania. 6. ed. São Paulo: Papirus. 2007.

BRASIL. Constituição da República Federativa do Brasil, de 05 de outubro de 1988. Brasília, 1988. Diário Oficial da União. Disponível em: http://www.planalto.gov.br/ccivil_03/Constituicao/Constituicao.htm Acesso em: julho.2021.

BRASIL. Decreto № 5.296 de 2 de dezembro de 2004. Diário Oficial da União, 3 dez. 2004. Disponível em: http://www.planalto.gov.br/ccivil_03/_ato20042006/2004/decreto/d5296.htm . Acesso em: 25 jul. 2021.

BRASIL. Lei n. 13.105, de 16 de março de 2015. Disponível em: <https://www.planalto.gov.br/ccivil_03/_ato2015-2018/2015/lei/l13105.htm> Acesso em: jul.2021.

BRASIL. Lei no 10.406, de 10 de janeiro de 2002. Institui o Código Civil. Diário Oficial da União: seção 1, Brasília, DF, ano 139, n. 8, p. 1-74, 11 jan. 2002.

BRASIL. Lei n‥ 10.048, de 08 de Novembro de 2000. Diário Oficial [da República Federativa do Brasil], Brasília. Disponível em: https://www.presidencia.gov.br/ccivil_03/Leis/L10048.htm . Acesso em: 30jul.2021.

BRASIL. Lei no. 10.098, de 19 de Dezembro de 2000. Diário Oficial [da República Federativa do Brasil], Brasília. Disponível em: https://www.presidencia.gov.br/ccivil_03/Leis/L10098.htm . Acesso em: 30jul.2021.

RC: 97618

Verfügbar in: https://www.nucleodoconhecimento.com.br/gesetz/prioritaetsdienst 
BRASIL. Presidência da República. Lei 7.853 de 24 de outubro de 1989. Disponível em:http://www.planalto.gov.br/ccivil_03/leis/l7853.htm - Acesso em 26/07/2021.

CARTA Mundial do Direito à Cidade. Fórum Social das Américas - Quito - Julho 2004; Fórum Mundial Urbano - Barcelona - Setembro 2004; V Fórum Social Mundial - Porto Alegre - Janeiro 2005. Disponível em: Acesso em: 30 jul. 2021.

CARVALHO, Afrânio de. Registro de imóveis. Rio de Janeiro: Editora Forense, 2011.

CENEVIVA, Walter. Lei dos notários e registradores comentada. São Paulo: Saraiva, 2008.

CLEMENTE, Carlos Aparício. Lei de cotas para o trabalho de pessoas com deficiência: Análise e fundamentação dos principais argumentos favoráveis e contrários ao seu cumprimento. 2015.

GALIANI, Luiz Antônio. Os princípios basilares do fólio real. RJ n. 212, jun/95.

GOFFMAN, Erving. Estigma: notas sobre a manipulação da identidade deteriorada. 4ㄹ. ed. Rio de Janeiro:LTC, 2008.

GUGEL, Maria Aparecida. Benefício da prestação continuada e trabalho: Mudanças da Lei $N^{\circ} 12.470$, de 31 de agosto de 2011. Disponível em: Acesso em jul.2021.

KOYAMA, D. B. Os reflexos da lei 13.146/2015 - Estatuto da Pessoa com Deficiência - no sistema jurídico brasileiro. Universidade Presbiteriana Mackenzie, São Paulo, 2017.

LAGO, Ivan Jacopetti do. O atendimento prioritário da Lei Federal 13.146/2015 (Estatuto da Pessoa Com Deficiência) e o princípio da prioridade do registro de imóveis.Revista de Direito Imobiliário,São Paulo, ano 39, v.80, jan-jun.2016.

$\mathrm{RC}: 97618$

Verfügbar in: https://www.nucleodoconhecimento.com.br/gesetz/prioritaetsdienst 
LORENTEZ, Lutiana Nacur. A norma da igualdade e o trabalho das pessoas portadoras de deficiência. São Paulo: LTr, 2006.

MAINIERI, Tiago; ROSA, Elisa Costa Ferreira. Comunicação pública, cidadania e democracia-algumas reflexões. Revista Comunicação Midiática, v. 7, n. 1, p. p. 192-206, 2012.

ORGANIZAÇÃO DAS NAÇÕES UNIDAS (ONU). Declaração de Direitos do Deficiente Mental - 1971. Aprovada pela resolução n. A/8429 da Assembleia Geral da ONU de 22 de dezembro de 1971. Disponível em: http://www.direitoshumanos.usp.br/index.php/Direito-dos-Portadores-deDefici\%C3\%AAncia/declaracao-de-direitos-do-deficiente-mental.html. Acesso em 27/07/2021.

SILVA, C. H. R. Teoria das Incapacidades. [online]. 2008. Disponível em: <http://www.ribeirodasilva.pro.br/teoriadasincapacidades.html> Acesso em: jul.2021.

VASCONCELOS, Julenildo Nunes; CRUZ, Antônio Augusto Rodrigues. Direito notarial: teoria e prática. [S.I.] Juarez de Oliveira, 2000.

\section{ANHANG - REFERENZFUßNOTE}

4. Reihe von rechtlichen Maßnahmen zum Schutz von Situationen, die sich im Aufbau, der Änderung oder dem Löschen befinden.

5. Vorgeschlagene rechtliche Maßnahmen zur Beierung etablierter Situationen

6. Sie legt allgemeine Standards und grundlegende Kriterien zur Förderung der Barrierefreiheit für Menschen mit Behinderungen oder eingeschränkter Mobilität fest und sieht weitere Maßnahmen vor.

7. Es regelt die Gesetze in 10.048 vom 8 . November 2000, die der Betreuung von Menschen Vorrang einräumen, und 10.098 vom 19. Dezember 2000, die allgemeine

RC: 97618

Verfügbar in: https://www.nucleodoconhecimento.com.br/gesetz/prioritaetsdienst 
Standards und grundlegende Kriterien für die Förderung der Zugänglichkeit für Menschen mit Behinderungen oder eingeschränkter Mobilität festlegen und andere Maßnahmen vorsieht.

8. Es räumt der Betreuung der von ihm festgelegten Menschen Priorität ein und sieht andere Maßnahmen vor.

Eingereicht: Juli 2021.

Genehmigt: September 2021.

RC: 97618

Verfügbar in: https://www.nucleodoconhecimento.com.br/gesetz/prioritaetsdienst 